\title{
Anterior Cruciate Ligament Injuries and Outcome of Arthroscopic Reconstruction Using Quadrupled Hamstrings: A Kenyan Experience
}

\author{
T.K. Byakika, MBChB, MMed (Surg), \\ Consultant Orthopaedic Surgeon, P.O. Box 76376, Nairobi, Kenya
}

\begin{abstract}
Objective: To study the results of arterior cruciate ligament (ACL) repairs using arthroscopic assisted reconstructions using harmstrings. A follow-up rehabilitation programme of immediate mobilisation, weight bearing and extension.
\end{abstract}

Subjects: Twenty arthroscopic reconstructions of the anterior cruciate ligament using the hamstrings were performed at the Nairobi hospital between October 2004 and December 2006.

Methodology: A consecutive study of 20 patients whose clinical work up and surgery were done by the author and assesed post-operatively for pain stability and satisfaction. Complications noted are evaluated and addressed accordingly.

Setting: The Nairobi Hospital.

Results: All the patients had satisfactory results based on patient satisfaction, freedom from pain and range of movements.

Conclusion: Arthroscopically assisted hamstring ACL repair has good results and is to be encouraged.

\section{INTRODUCTION}

Anterior Cruciate Ligament $(\mathrm{ACL})$ injuries are on the increase with the greater interest in sports and previously active life skills (1). Loss of the anterior cruciate ligament was not considered important to the long term function of the knee (2). However several studies now show that instability after rupture of the ACL may lead to recurrent episodes of knee giving way, risk of meniscal injury and premature degenerative changes $(3,4,5)$.

There are many different methods that have been used to restore stability to the ACL-deficient knee but several have produced poor long term results. These include direct repair, extra-articular procedures $(6,7)$ and intra-articular prostheses (8-10).

The two most commonly used autografts for intraarticular reconstruction of the $A C L$ are the combined semi-tendinosus and gracilis tendons (STG) and central one-third of the patella tendon (PT).

Though PT autografts were at one time considered the gold standard (6) they have shown no superiority to the hamstring graft $(6,9,11)$. The hamstring method may be advantageous in that there are no anterior knee pains as in the PT method and also there is less loss of extension $(6,9)$. In this study, the hamstrings were used to reconstruct the $A C L$, however the emphasis is on the arthroscopic technique and its advantages (12).

\section{MATERIALS AND METHODS}

Between 0ctober 2004 and December 2007, 20 patients with torn anterior cruciate ligaments were seen and operated upon arthroscopically using quadrupled hamstrings.

All the 20 patients were attended to by the author. A detailed history was obtained and a clinical examination of the knee performed. In 13 cases (65\%) an MRI scan was performed to confirm the diagnosis. In seven cases (35\%) the diagnosis was confirmed at arthroscopy. In all the cases the patients were admitted to hospital (18 Nairobi hospital) two (others).The patients then underwent arthroscopic reconstruction of the $A C L$ using hamstrings.

At surgery under general or spinal anaesthesia, with a tourniquet inflated to $350 \mathrm{mmHg}$ the semi-tendinosus was harvested through a $5 \mathrm{~cm}$ incision over the pes anserinus with a tendon stripper. Two tendons the 
gracilis and semi-tendinosus in that order were then prepared by a trained assistant nurse. Whip stitches were then put in on either end and then a quadruple arrangement made. Fixation was by an endobutton proximally and Bio $\mathrm{RCl}$ screws distally.

The reconstructive procedure was undertaken arthroscopically where by the remnants of the anterior cruciate ligament were removed and the tibial tunnel made using the acufex tibial guide, the guidelines for the tibial tunnel placement were the anterior horn of the lateral meniscus and the posterior cruciate ligament, the guide tip just hugged the PCL.The femoral tunnel was placed posteriorly right behind the so called (resident's ridge), with the knee at 90 degrees of flexion. On the right side this was at 110'clock and on the left at 1 o'clock. The placement of the femoral tunnel was paramount to success of the procedure. Notch-plasty was not done in any of the cases. The knee was taken through at least 30 cycles after the endobutton was in place. The Bio $\mathrm{RCl}$ screw was then inserted in the tibial tunnel with maximum tension on the graft and the knee at 30 degrees of extension. After the graft was in place, impingement was not noted in all the cases.

Immediately after surgery a hinged knee brace was applied and mobilization with range of motion exercises commenced on the next day. Full weight bearing was allowed in all patients. At day 4 or 5 all the patients were discharged, ambulant on crutches and had minimal pains.

Then followed a 3-9 months period of supervised physiotherapy and regular visits in the outpatient clinic. The same rehabilitation protocol was used for all the patients though at different physiotherapy centers. Namely closed chain exercises and range of movement training from the first postoperative day. Open chain exercises were allowed after 12 weeks. Running was allowed after 12 weeks and a resumption of contact sports no earlier than eight months.

The out come of the surgical procedure is evaluated on the basis of absence of pain and instability with a return to normal pre accident activities.

\section{RESULTS}

Of the 20 patients 15 were males and 5 females. The mean age was 33 years with the 18 to 30 group having the larger number (10 patients). Sports injuries were the commonest cause of these ruptures 11 patients (55\%), with assault and other forms of trauma accounting for nine $(45 \%)$. In one case there was no history of prior knee trauma. Sports injuries all occurred at an early age, less than 30 years even if some of the patients presented later in life.

In 10 (55\%) cases, the patients presented early, less than six months and in $10(50 \%)$ there was at least one year duration from trauma to surgery. Eight (40\%) patients had associated meniscal tears, which were concomitantly treated by partial menisectomy at reconstruction or initial arthroscopy.

The complications seen in $10(50 \%)$ of the patients were as follows two tendon amputations at harvesting, one septic arthritis which was successfully treated with arthroscopic lavage and antibiotics, three cases of limited range of motion, one deep vein thrombosis, one extrusion of screw and two mild laxity of the knee.

Assessment was made in all patients at the short term, less than six months and the long term, more than six months where applicable as most of these patients were operated in the last half of last year.

For the outcome, the chosen criteria was knee pain, and a direct question do you feel better after the reconstruction or regret it, stability of the knee and return to pre-injury status. All patients (100\%) reported no pain and said their knees were stable, by six months post-operatively. Of the two competitive soccer players the one operated in December 2004 had returned to his goal keeping with a national club, and the other operated in July 2006 was on the verge of returning to at least soccer training. Laxity was however noted among these two football players. All the patients at whatever duration of their rehabilitation said they felt better after the surgery and none regretted it. 
Table 1

Results

\begin{tabular}{lc}
\hline $\begin{array}{l}\text { No pain } \\
\text { Do you regret your operation } \\
\text { Yes }\end{array}$ & 20 \\
$\quad$ No & None (0\%) \\
Knee Stability & $20(100 \%)$ \\
Negative Lachman & 20 \\
Negative lateral Pivot shift test & 20 \\
Range of Motion & 15 \\
$\begin{array}{l}\text { Full range of motion by at least } 6 \text { months } \\
\text { Return to pre accident activities } \\
\text { At least } 1 \text { year follow up }\end{array}$ & 9 out of 9 \\
\end{tabular}

Table 2

Duration of hospital stay

3 - 5 days 20 patients

Most of the patients left on day 4 and none stayed beyond 5 days.

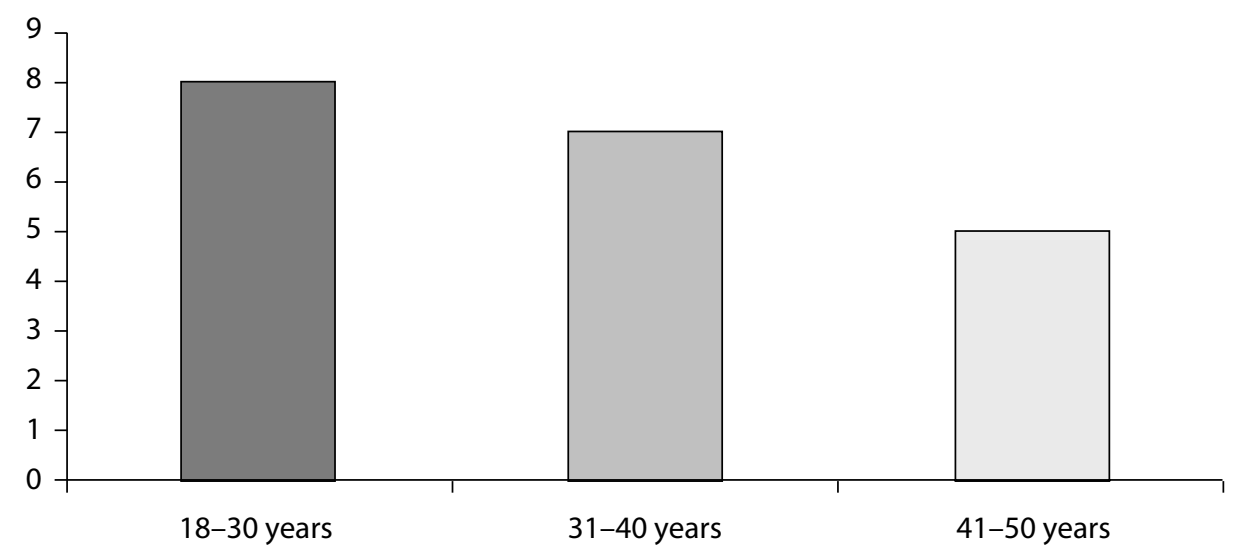

Figure 1: Age distribution

The average age was 33 years. The age range was $18-50$ years. The commonest age group in this study was $18-30$ years variance with the other studies. 


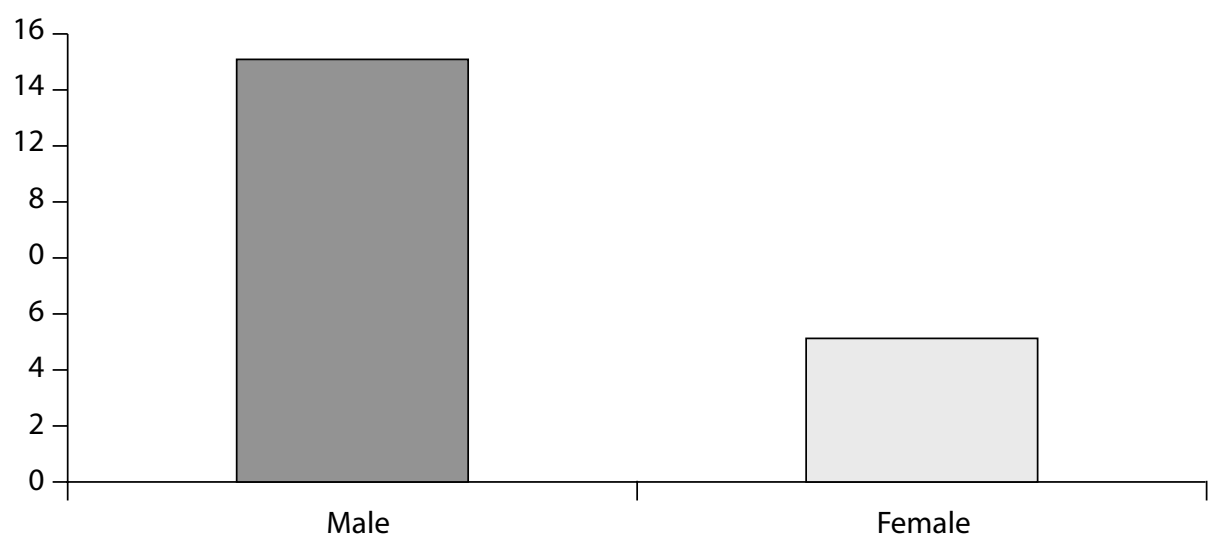

Figure 2: Sex distribution

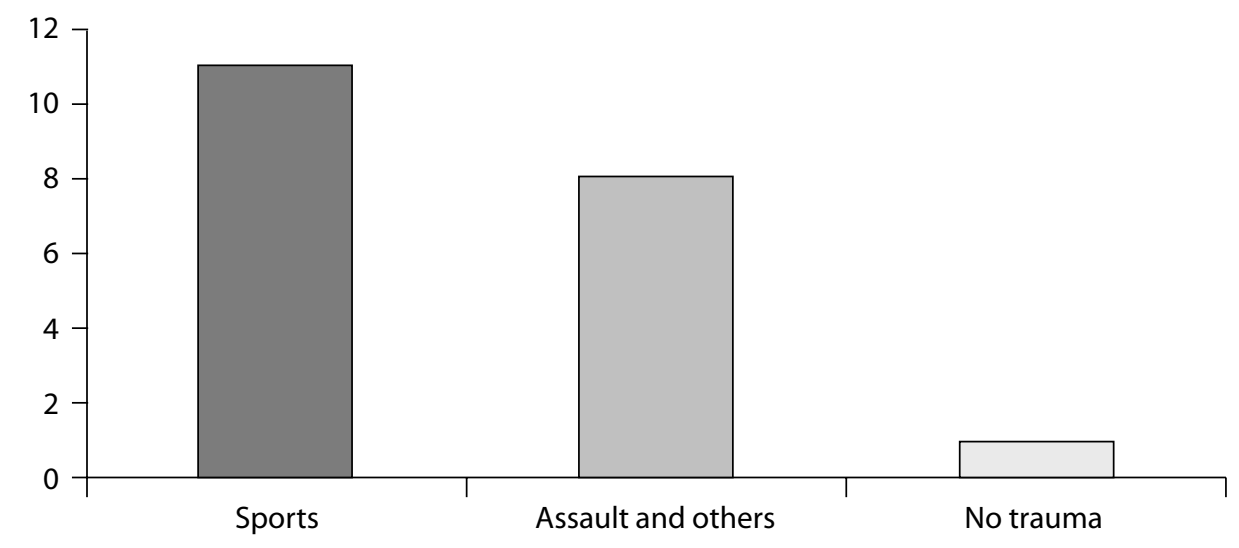

Figure 3: Aetiology

Sports injuries were as common as assault and other causes.

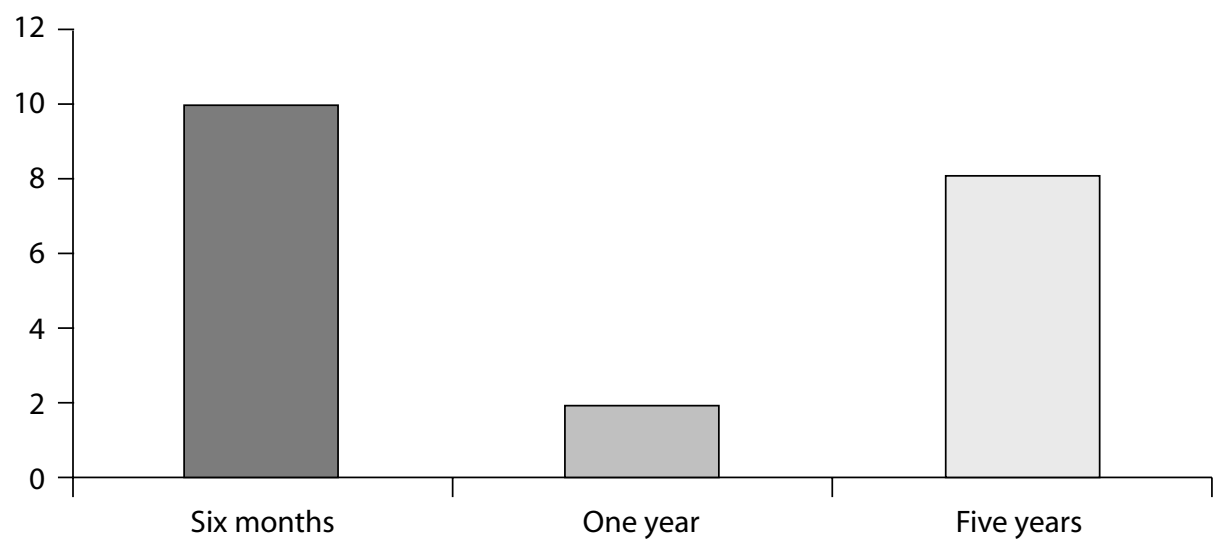

Figure 4: Duration from trauma correction 


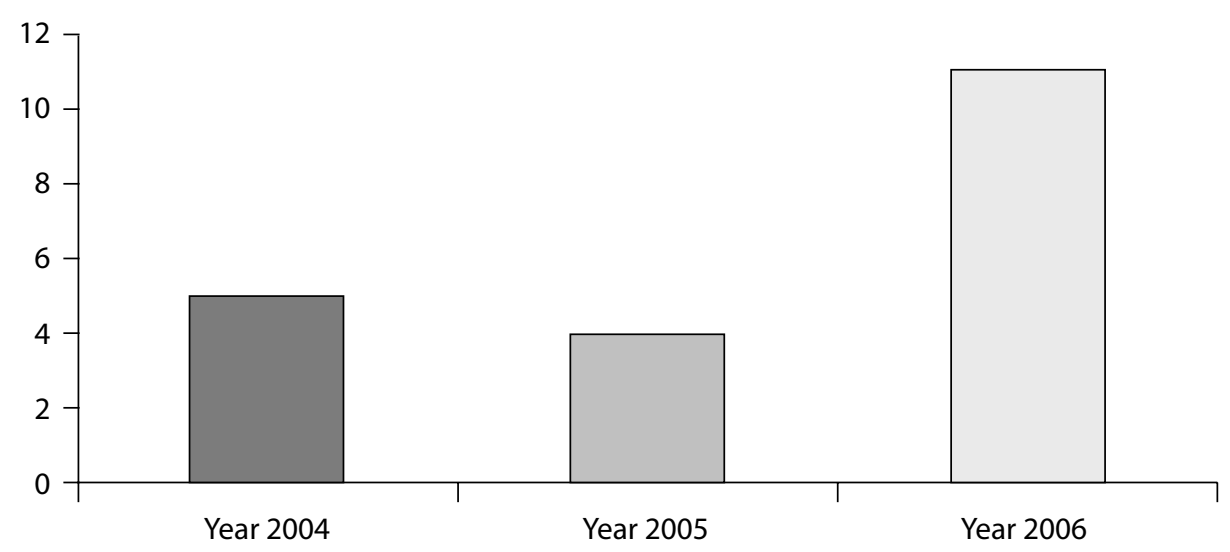

Figure 5: Number of cases operated

Table 3

Complications

\begin{tabular}{lc}
\hline Operative & 2 \\
Tendon amputation & \\
Post operative & 1 \\
Infection & 3 \\
Limitation of range of motion & 1 \\
DVT & 1 \\
Extrusion of screw & 2 \\
Laxity (Mild) & 10 \\
\hline Total & \\
\hline
\end{tabular}

\section{DISCUSSION}

The number of patients in this study who had suffered anterior cruciate ligament $(\mathrm{ACL})$ tears due to sports injuries was 11 (55\%), trauma and other causes accounting for nine $(45 \%)$ of cases. This is a variance with other studies where sports trauma accounts for over $80 \%$ of causes (7). This is probably due to a higher level and wider participation of the populations in sports in the developed countries. It may also be that a lot of our patients are remaining undiagnosed and hence we are missing out on the sports related injuries. This is brought out in the study where eight (40\%) patients had chronic ACL tears that had remained undiagnosed.

There were 15 males (75\%) and five females (25\%), with the age range being 18 to 50 years. This is at variance with other studies $(6,7,9)$ where the sex ratio is almost 1:1. In our environment however trauma related injuries are commoner among males. The mean age of 33 was also higher than that of 25 years in one study (7), but was similar in another one (13). In two cases the $\mathrm{ACL}$ reconstructions were done and 14 and 15 years after trauma which contributed to a higher population study age.

The criteria for performing $\mathrm{ACL}$ reconstructions were pain and instability in the knee and all 20 patients had that in this study. The main aim of performing ACL reconstructions is to restore stability, allow patients to return to the pre-accident activities and prevent the occurrence of meniscal injuries and degeneration of the joint $(2-4,12,14)$. One may therefore have a torn $A C L$ and not require reconstruction if it is asymptomatic, in this study all the patients met the criteria for reconstruction. Seven (77\%) out of nine patients in the chronic ACL tear group had meniscal tears with two of these over 45 
years having osteoarthritis changes. This percentage is very high in comparison with other findings elsewhere, $(1-4,14,15)$. These patients had remained undiagnosed and lived with unstable knees thus leading to a high prevalence of tears. This could have been prevented by early diagnosis and intervention to reconstruct the ACL. Moreover the patients with associated injuries such as meniscal tears, chondral damage, etc don't do as well as the ones with isolated tears of the ACL despite reconstruction so it is very important to catch them early and do the reconstruction. A higher index of suspicion among knee injured patients is therefore required in our setting.

The method of $A C L$ reconstruction here was arthroscopic with the hamstrings. There is also the option of the bone-patellar tendon-bone graft. $(9,13)$. Comparative studies done $(6,9,11,13)$, showed no difference in the subjective and objective assessments, the only significant differences involved the ability to kneel and normalize hyperextension with better results in the semitendinosus group, with the absence of anterior knee pain.

The placement of the tibial and femoral tunnels is critical to the success of the procedure more especially the femoral tunnel and this can be suitably placed with the endoscopic technique (6). In this study the femoral tunnel was placed well behind the residents' ridge just anterior to the posterior edge of the femoral condyle.

The endoscopic reconstruction technique however is technically demanding, with a long learning curve and requires that one has all or most of the equipment. Until very recently hospitals in our country have been very reluctant to invest in arthroscopic equipment making for a late start in these techniques that are so routinely performed in the developed countries. The situation has been one of some visiting surgeons carrying their equipment and carrying out the procedures here. About four years ago patients opted to be referred abroad instead of having the surgery performed locally, fortunately this scenario is changing as evidenced by the increased number of reconstructions that were performed, 11 in 2006.

The complications of surgery noted in this study were ten and compared favourably with those in other studies $(7,16)$. Two patients had mild laxity which is reported in all series where hamstrings have been used $(6,10,13)$. It is indeed one of the shortcomings sited when this method of reconstruction is used but it does not seem to affect knee function and stability. Deep vein thrombosis is very rare and so was indeed unusual when it occurred in the one patient in this study. Tendon amputation occurred in two cases earlier on in the work, though it is a well documented complication (6). It is avoided by freeing the tendon of its adhesions and using a good tendon stripper.

The main advantages of arthroscopic reconstruction is the minimal morbidity to the patients the subsequent short duration of stay maximum being five days in this study. A short hospital stay is cost effective and also more acceptable to the patients. This has been extended to outpatient or day surgery in some centers in the U.S.A (17).

The short follow up of at least six months show promising results with all the patients reporting no pains and stable knees. Also none of them regretted the procedure. In four of the patients when physiotherapy was not followed or was inadequately performed the results were inferior. Physiotherapy does indeed contribute significantly to good results $(8,9,18,19)$.

In conclusion since October 2004, 20 patients with symptomatic tears of the anterior cruciate ligaments were surgically treated with arthroscopic reconstruction using quadrupled hamstring tendons. The results so far have been good with no failures. This is a good and cost effective method of reconstruction that can be used on our patients with symptomatic torn anterior cruciate ligaments.

\section{REFERENCES}

1. Hughstone J.C. Anterior cruciate deficient knee. Amer. J. Sports Med. 1983; 11: 1-2.

2. McDaniel W.J. and Dameron T.B. Untreated ruptures of the anterior cruciate ligament. J. Bone Joint Surg.[Amer]. 1980; 62A: 696-705.

3. Arnold J.A., Coker T.P., Heaton L.M., Park J.P. and Harris W.D. Natural history of anterior cruciate tears. Amer. J. Sports Med. 1979; 7: 305-313.

4. Noyes F.R., Mooar P.A., Matthews D.S. and Butler D.L. The symptomatic anterior cruciate deficient knee Part 1. The longterm functional disability in athletically active individuals. J. Bone Joint Surg. [Amer.]. 1983; 65A: 154-162.

5. Johnson R.J., Beynnon B.D., Nichols C.E. and Renstrom P.A. Current concepts review: The treatment of injuries of the anterior cruciate ligament. J. Bone Joint Surg. [Amer.]. 1992; 74-A: 140-151.

6. Ericksson K., Anderberg P., Hamberg P., Logfren A.C., Bredneberg M., Westman I. and WredmarkT. A comparison of quadruple semitendinosus and patellar tendon grafts 
in reconstruction of the anterior cruciate ligament. J. Bone Joint Surg. [Brit.]. 2001; 83: 348-354.

7. Webb J.M., Ian S.I., Corry S., et al. Endoscopic reconstruction for isolated anterior cruciate ligament rupture.J. Bone Joint Surg. [Brit.]. 1998; 80-B: 288-294.

8. Harter R.A., Osternig L.R., Singer K.M., et al. Longterm evaluation of knee stability and function following surgical reconstruction for anterior cruciate ligament insufficiency. Amer. J. Sports Med. 1988; 16: 434-443.

9. Aune A.K., Holm I., et al. Four strand hamstring tendon autograft compared with patellar tendon autograft for anterior cruciate ligament reconstruction. Amer. J. Sports Med. 2001; 29: 722-728.

10. Paolo Aglietti et al. J. Bone Joint Surg. [Amer.]. 2004; 86-A: 2143-2153.

11. Steiner M.E., Hecker A.T., Brown C.H. Jr and Hayes W.C. Anterior cruciate graft fixation: comparison of hamstring and patellar tendon grafts. Amer. J. Sports Med. 1994; 22: 659-656.

12. Dandy D.J., Flanagan J.P. and Steenmeyer V. Arthroscopy and the management of the ruptured anterior cruciate ligament. Clin. Orthop. 1982; 167: 43-49.

13. Williams R.J., Jon H., Petrigliano F., et al. Anterior cruciate ligament reconstruction with a four strand hamstring tendon autograft. J. Bone Joint Surg. [Amer.]. 2004; 86: 225-232.

14. Kannus P. and Jarvinen M. Conservatively treated tears of the anterior cruciate ligament: Long-term results. J. Bone Joint Surg. [Amer.]. 1987; 69A: 1007-1012

15. Hawkins R.J., Moisamore G.W. and Merrit T.R. Follow up of the acute non operated isolated anterior cruciate ligament tear. Amer. J. Sports Med. 1986; 14: 205-210.

16. Kartus J., Magnusson L., Stener S., et al. Complication following arthroscopic anterior ligament reconstruction: A 2-5 year follow up of 604 with a special emphasis on anterior knee pain. Knee Surg. Sports Traumatol. Arthscop. 1999; 7: 2-8.

17. Siegel M.G. and Barber-Westin S.D. Arthrscopic-assisted outpatient anterior cruciate ligament reconstruction using the semitendinosus and gracilis tendons. Arthroscopy. 1998; 14: 268-277.

18. Shelbourne K.D. and Nitz P. Accelerated rehabilitation after anterior cruciate ligament reconstruction. Amer. J. Sports Med. 1990; 18: 292-299.

19. Muneta T., Sekiya I., Oguichi T., et al. Effects of aggressive early rehabilitation on the outcome of anterior cruciate ligament reconstruction with multi strand semitendinosus tendon. Int. Orthop. 1998; 22: 352-356. 